\title{
Synthesis of 4-tert-butyl-2-(thiomorpholin-4-ylmethyl)phenol, and 4-tert-butyl-2,6-bis(thiomorpholin-4-ylmethyl)phenol
}

A. Ma. Velázquez ${ }^{1}$, L.Torres ${ }^{1}$, R.González ${ }^{1}$, I.Martínez ${ }^{1}$, A.Valencia ${ }^{1}$, A.Pecina ${ }^{1}$, L.Torres, I.Menconi ${ }^{1}$, L.Martínez ${ }^{1}$, A.Ramírez ${ }^{1}$, R.Hernández ${ }^{1}$, R.López-Castañares ${ }^{2}$, O.Olvera-Neria ${ }^{3}$, E.Angeles**1,

1) Facultad de Estudios Superiores Cuautitlán, Universidad Nacional Autónoma de México,

2) Facultad de Química de la UAEM,

3) Instituto de Ciencias Básicas e Ingenieria, Universidad Autónoma del Estado de Hidalgo

e-mail: angeles@,servidor.unam.mx

Received: 2 April 2004 / Accepted: 31 May 2004 / Published: 1 July 2005

Keywords: phenol, thiomorpholine

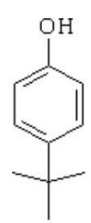

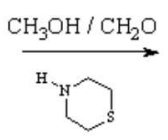

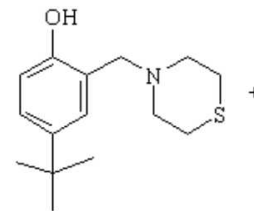

2

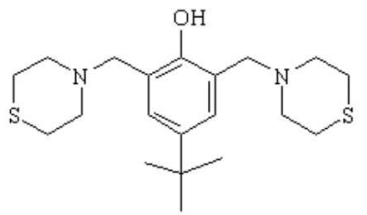

3

4-tert-butyl-2-(thiomorpholin-4-ylmethyl)phenol (2) and 4-tert-butyl-2,6-bis(thiomorpholin-4-ylmethyl)phenol (3) were prepared from 4- tert-butylphenol (1) and thiomorpholine and formaldehyde $(37 \%)$ in methanol as solvent. A solution of methanol $(50 \mathrm{~mL})$ and 4-tert-butylphenol $(1.49 \mathrm{~g}, 9.92 \mathrm{mmol}) 1$ was prepared and and heated at $40{ }^{\circ} \mathrm{C}$ for 15 minutes, after that a solution of thiomorpholine $(2.0 \mathrm{~g}, 20.7 \mathrm{mmol})$ and formaldehyde $(1.50 \mathrm{~mL}, 20.15 \mathrm{mmol})$ in methanol were added. When the addition was completed, the reaction mixture was stirred at reflux for $24 \mathrm{hrs}$. The solvent was eliminated using rotavapor and reaction mixture was poured into water and extracted with ethyl acetate. Chromatography on silica gel (30/70 EtOAc/ $n$-hexane) afforded two crystalline products $\mathbf{2}$ and $\mathbf{3}$ (5\% and $35 \%$ yield).

\section{4-tert-butyl-2-(thiomorpholin-4-ylmethyl)phenol (2)}

Melting Point: 85-87 $\mathrm{C}^{\circ}$ (methanol, uncorrected).

IR $\left(\mathrm{CHCl}_{3}\right.$ film; $\left.\mathrm{cm}^{-1}\right): 3456(\mathrm{O}-\mathrm{H}) ; 3197\left(\mathrm{C}_{\mathrm{sp} 2}-\mathrm{H} \mathrm{Ar}\right) ; 2886\left(\mathrm{C}_{\mathrm{sp} 3}-\mathrm{H}\right)$.

${ }^{1} \mathrm{H}-\mathrm{NMR}\left(300 \mathrm{MHz} ; \mathrm{CDCl}_{3}\right): \delta=10.33(1 \mathrm{H}, \mathrm{s}, \mathrm{OH}) ; 7.18(1 \mathrm{H}, \mathrm{dd}, J=8.4 \mathrm{~Hz}, 2.7 \mathrm{~Hz}) ; 6.94(1 \mathrm{H}, \mathrm{d}, J=2.7 \mathrm{~Hz}) ; 6.74(1 \mathrm{H}, \mathrm{d}, 8.4 \mathrm{~Hz}) ; 3.70(2 \mathrm{H}, \mathrm{s}, \mathrm{Ar}-\mathrm{CH} 2) ; 2.82(4 \mathrm{H}, \mathrm{m},-\mathrm{S}-\mathrm{CH} 2-)$; $2.71\left(4 \mathrm{H}, \mathrm{m},-\mathrm{N}-\mathrm{CH}_{2}-\right) ; 1.27\left(9 \mathrm{H}, \mathrm{CH}_{3}\right)$.

${ }^{13} \mathrm{C}-\mathrm{NMR}\left(75 \mathrm{MHz} ; \mathrm{CDCl}_{3}\right): \delta=155(\mathrm{C}) ; 141.8(\mathrm{C}) ; 125.60(\mathrm{CH}) ; 125.49(\mathrm{CH}) ; 119.77(\mathrm{C}) ; 115.47(\mathrm{CH}) ; 62.51(\mathrm{Ar}-\underline{\mathrm{CH}} \underline{2}) ; 54.36(-\mathrm{N}-\mathrm{CH} 2-) ; 33.84(\mathrm{C}) ; 31.48(\mathrm{CH} 3) ; 27.79$ $\left(-\mathrm{S}-\mathrm{CH}_{2}-\right)$.

MS (FAB; m/z, \%): 266(80\%); $265(100 \%) ; 163(45 \%)$.

Elemental Analysis: Calculated for $\mathrm{C}_{15} \mathrm{H}_{23} \mathrm{NOS}$ : C, 67.88\%; H, 8.73\%; N, 5.28\%; O, 6.03\%; S, 12.08\%. Found: C, 67.58\%; H, 8.75\%; N, 5.41\%; O, 6.09\%; $\mathrm{S}, 12.01 \%$.

\section{4-tert-butyl-2,6-bis(thiomorpholin-4-ylmethyl)phenol (3)}

Melting Point: $95-97 \mathrm{C}^{\circ}$ (methanol, uncorrected).

IR ( $\mathrm{CHCl}_{3}$ film; $\left.\mathrm{cm}^{-1}\right): 3403(\mathrm{O}-\mathrm{H}) ; 3089\left(\mathrm{C}_{\mathrm{sp} 2}-\mathrm{H} \mathrm{Ar}\right) ; 2986\left(\mathrm{C}_{\mathrm{sp} 3}-\mathrm{H}\right)$.

${ }^{1} \mathrm{H}-\mathrm{NMR}\left(300 \mathrm{MHz} ; \mathrm{CDCl}_{3}\right): \delta=10.69(1 \mathrm{H}, \mathrm{s}, \mathrm{OH}) ; 7.09(2 \mathrm{H}, \mathrm{s}) ; 3.71\left(4 \mathrm{H}, \mathrm{s}, \mathrm{Ar}-\mathrm{CH}_{2}\right) ; 2.86\left(8 \mathrm{H}, \mathrm{m},-\mathrm{S}-\mathrm{CH}_{2}-\right) ; 2.76\left(8 \mathrm{H}, \mathrm{m},-\mathrm{N}-\mathrm{CH}_{2}-\right) ; 1.27(9 \mathrm{H}, \mathrm{CH} 3)$.

${ }^{13} \mathrm{C}-\mathrm{NMR}\left(75 \mathrm{MHz} ; \mathrm{CDCl}_{3}\right): \delta=153.6(\mathrm{C}) ; 141.14(\mathrm{C}) ; 125.79(\mathrm{CH}) ; 121.22(\mathrm{C}) ; 58.81\left(\mathrm{Ar}_{-} \underline{\mathrm{CH}_{2}}\right) ; 54.42\left(-\mathrm{N}-\mathrm{CH}_{2}-\right) ; 33.78(\mathrm{C}) ; 31.47(\mathrm{CH} 3) ; 27.74(-\mathrm{S}-\mathrm{CH} 2-)$.

MS (FAB; m/z, \%): $381(35 \%) ; 278(100 \%) ; 175(50 \%)$.

Elemental Analysis: Calculated for $\mathrm{C}_{20} \mathrm{H}_{32} \mathrm{~N}_{2} \mathrm{OS} 2$ : C, 63.11\%; H, 8.47\%; N, 7.36\%; O, 4.20\%; S, 16.85\%. Found: C, 63.42\%; H, 8.51\%; N, 7.29\%; O, 4.25\%; S, 16.91\%.

Acknowledgements

The authors wish to acknowledge to PAPIIT/UNAM Projects No IN205902 and IN207705 and ALPHARMA SA de CV, by partially support this work. We would like to thank C.Barajas, F.Sotres, P.García, D.Jiménez from FESC-UNAM and Rosa I.del Villar M., Oscar Yañez and Georgina Duarte from USAI-UNAM for their skillful technical assistance and DGSCA-UNAM for their support. As a part of Project Cátedra Química Medicinal of FESC-UNAM.

\section{References}

1. Biava, M., Fioravanti, R., Porretta, G.C., Deidda, D., Maullu, C., Pompei M. Biorg.\& Med. Chem. Lett. 1999, 9, $2983-2988$.

2. Teipel, S.; Griesar, K.; Haase, W.; Krebs, B. Inorganic Chemistry 1994, 33, 456-64.

3. Hodgkin, J.H., Aust .J. Chem., 1984, 37, 2371-2378. 

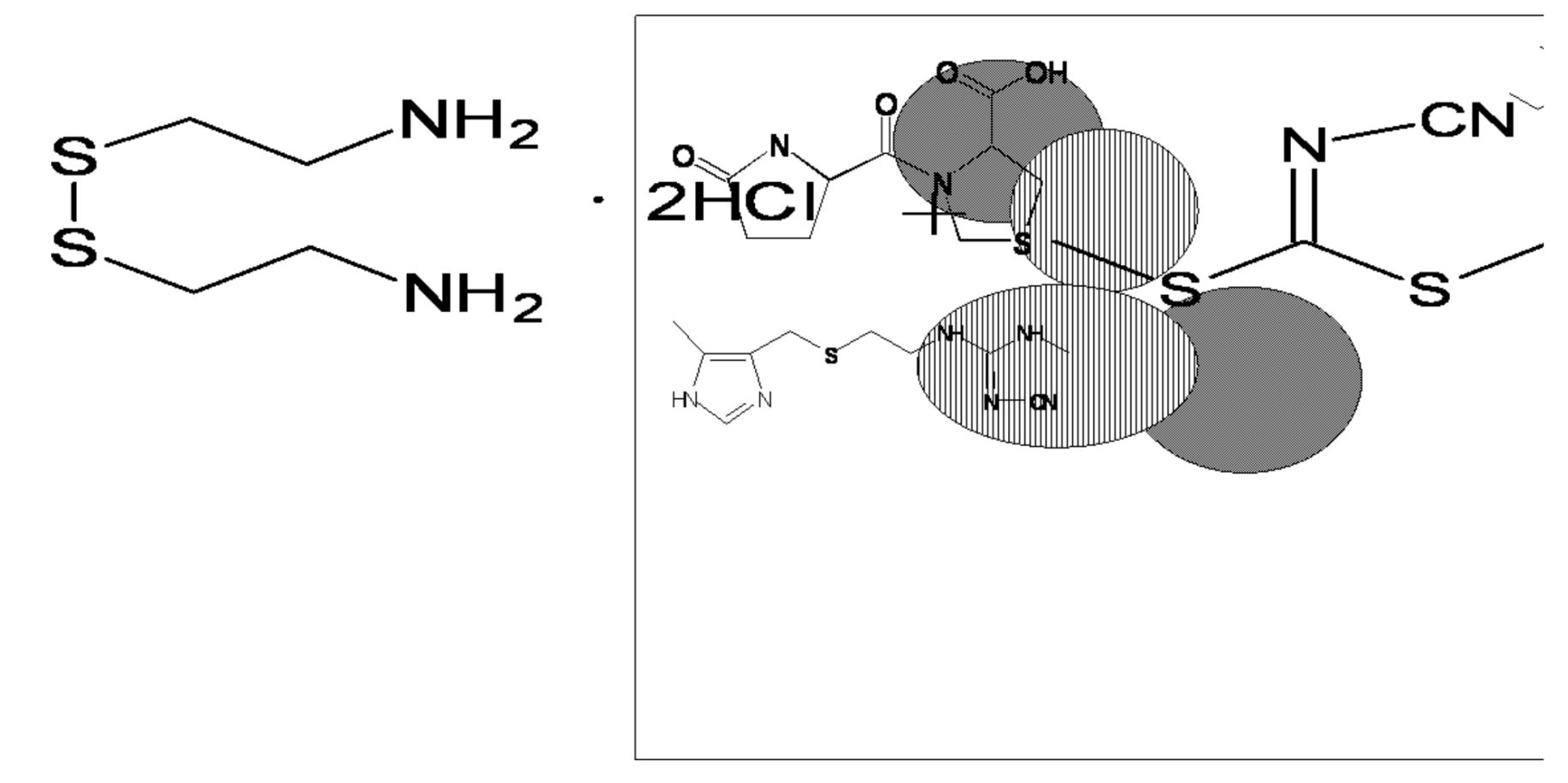

(C) 2005 MDPI. All rights reserved.

2005 MDPI. All rights reserved. 